\title{
AMPKa1 confers survival advantage of colorectal cancer cells under metabolic stress by promoting redox balance through the regulation of glutathione reductase phosphorylation
}

\author{
Ying-Nan Wang ${ }^{1} \cdot$ Yun-Xin $\mathrm{Lu}^{1} \cdot$ Jie Liu ${ }^{2} \cdot$ Ying $\mathrm{Jin}^{1,3} \cdot$ Hui-Chang $\mathrm{Bi}^{4} \cdot \mathrm{Qi}$ Zhao $\mathbb{1}^{1} \cdot$ Ze-Xian Liu $\mathbb{1}^{1} \cdot$ Ying-Qin $\mathrm{Li}^{2}$. \\ Jia-Jia Hu ${ }^{1} \cdot$ Hui Sheng ${ }^{1} \cdot$ Yi-Ming Jiang ${ }^{4} \cdot$ Chao Zhang $^{5} \cdot$ Feng Tian ${ }^{6} \cdot$ Yang Chen $^{1} \cdot$ Zhi-Zhong Pan $^{7} \cdot$ Gong Chen $^{7}$. \\ Zhao-Lei Zeng $\mathbb{1}^{1} \cdot$ Kai-Yan Liu $^{1} \cdot$ Marcia Ogasawara $^{8} \cdot$ Jin-Ping Yun $^{5} \cdot$ Huai-Qiang Ju $\mathbb{D}^{1} \cdot$ Jian-Xiong Feng ${ }^{1}$. \\ Dan Xie $\mathbb{I}^{1} \cdot$ Song Gao $\mathbb{1}^{1} \cdot$ Wei-Hua Jia ${ }^{1} \cdot$ Scott Kopetz ${ }^{6} \cdot$ Rui-Hua Xu $\mathbb{1}^{1,3} \cdot$ Feng Wang ${ }^{1,3}$
}

Received: 5 January 2019 / Revised: 9 August 2019 / Accepted: 15 August 2019 / Published online: 17 September 2019

(c) The Author(s) 2019. This article is published with open access

\begin{abstract}
Patients with stage II or III colorectal cancer (CRC) exhibit various clinical outcomes after radical treatments. The 5-year survival rate was between 50 and $87 \%$. However, the underlying mechanisms of the variation remain unclear. Here we show that AMPK $\alpha 1$ is overexpressed in CRC patient specimens and the high expression is correlated with poor patient survival. We further reveal a previously unrecognized function of AMPK $\alpha 1$, which maintains high level of reduced glutathione to keep reduction-oxidation reaction (redox) homeostasis under stress conditions, thus promoting CRC cell survival under metabolic stress in vitro and enhancing tumorigenesis in vivo. Mechanistically, AMPK $\alpha 1$ regulate the glutathione reductase (GSR) phosphorylation possibly through residue Thr507 which enhances its activity. Suppression of AMPK $\alpha 1$ by using nano-sized polymeric vector induces a favorable therapeutic effect, especially when in combination with oxaliplatin. Our study uncovers a novel function of AMPK $\alpha 1$ in redox regulation and identifies a promising therapeutic strategy for treatment of CRC.
\end{abstract}

These authors contributed equally: Ying-Nan Wang, Yun-Xin Lu, Jie Liu, Ying Jin

Supplementary information The online version of this article (https:// doi.org/10.1038/s41388-019-1004-2) contains supplementary material, which is available to authorized users.

Scott Kopetz

skopetz@mdanderson.org

$\triangle$ Rui-Hua Xu

xurh@sysucc.org.cn

$\triangle$ Feng Wang

wangfeng@ sysucc.org.cn

1 Sun Yat-sen University Cancer Center; State Key Laboratory of Oncology in South China, Collaborative Innovation Center for Cancer Medicine, Guangzhou 510060 Guangdong, China

2 Department of Biomedical Engineering, School of Engineering, Sun Yat-sen University, Guangzhou 510006 Guangdong, China

3 Department of Medical Oncology, Sun Yat-sen University Cancer Center, 510060 Guangzhou, China

\section{Introduction}

Colorectal cancer (CRC) is the third most prevalent cancer among males and the second most prevalent among females worldwide [1]. The incidence and mortality rates of CRC in China have been progressively increasing due to changes in lifestyle and diet [2-5]. Survival rates for CRC can vary

4 School of Pharmaceutical Sciences, Sun Yat-sen University, Guangzhou 510006 Guangdong, China

5 Department of Pathology, Sun Yat-sen University Cancer Center; State Key Laboratory of Oncology in South China, Collaborative Innovation Center for Cancer Medicine, Guangzhou 510060 Guangdong, China

6 Department of Gastrointestinal Medical Oncology, University of Texas MD Anderson Cancer Center, Houston, TX 77030, USA

7 Department of Colorectal Surgery, Sun Yat-sen University Cancer Center; State Key Laboratory of Oncology in South China, Collaborative Innovation Center for Cancer Medicine, Guangzhou 510060 Guangdong, China

8 Department of Experimental Therapeutics, University of Texas MD Anderson Cancer Center, Houston 77030 TX, USA 
based on a variety of factors, particularly clinical stage. The 5-year survival rate of patients with localized cancer (stage I) is $\sim 95 \%$. However, if the cancer has spread to distant parts of the body (stage IV), the 5-year survival rate is $13 \%$. For patients with stage II/III CRC, the prognosis varies even under similar treatments, with a 5-year survival rate between 50 and $87 \%$ [6]. Nevertheless, both the American Joint Committee on Cancer (AJCC) sixth edition and seventh edition staging systems do not address all survival discrepancies in CRC [7-9]. Therefore, determining other prognostic factors and underlying mechanisms for differences in survival are critical for making decisions about therapy.

AMP-activated protein kinase (AMPK) is a heterotrimeric serine/threonine protein kinase, consisting of $\alpha$ (catalytic), $\beta$ and $\gamma$ (regulatory) subunits, where phosphorylation of T172 in the $\alpha$-catalytic subunit is a critical event for its full activation [10]. AMPK has a central role in the regulation of cellular metabolism and energy homeostasis in mammalian tissues [11]. Once activated, AMPK maintains energy balance through the activation of catabolism to promote ATP production and the inhibition of anabolism to conserve ATP [12]. The role of AMPK in cancer has been hotly debated. AMPK had previously been regarded as a tumor suppressor, as evidenced by the demonstration that it negatively regulates aerobic glycolysis in cancer cells and suppresses tumor growth in vivo [13-15]. AMPK activators such as metformin have been shown to suppress tumor cell proliferation both in vitro and in vivo $[16,17]$. Deletion of the $\alpha 1$ catalytic subunit of AMPK accelerates the development of lymphoma in transgenic mice overexpressing $C-M Y C$ in B cells [13]. In contrast, AMPK is critical for promoting cancer cell survival under energy stress by maintaining NADPH levels [18]. AMPK also confers metabolic stress resistance to leukemia-initiating cells and promotes leukemogenesis [19]. Previous studies were mostly performed in cell lines and mouse models, it is important to identify the function of AMPK from a clinical standpoint and to clarify its underlying mechanism.

Here, we demonstrate that high levels of AMPK $\alpha 1$ are correlated with a poor prognosis in CRC patients and inhibition of AMPK $\alpha 1$ profoundly kills CRC cells by attenuating glutathione metabolism. In addition, we revealed that AMPK $\alpha 1$ regulates glutathione reductase phosphorylation possibly through residue Thr507 which enhances its activity. Downregulating the expression of AMPK $\alpha 1$ by using optimized poly(amine-co-esters)/short hairpin RNA (shRNA) polyplexes is able to significantly inhibit tumor growth in CRC mice models. Our results reveal the function of AMPK $\alpha 1$ and indicate that AMPK $\alpha 1$ can be a novel target in CRC.

\section{Results}

\section{AMPKa1 is significantly correlated with poor patient survival}

Using a reverse phase protein array (RPPA) assay, we quantified 141 cancer-relevant proteins and phosphoproteins in 134 paired samples from patients with stage II/III CRC. The clinical characteristics of those patients are summarized in Table S1. Univariate Cox regression analysis showed that 16 proteins were significantly correlated with overall survival (Table S2). Among those proteins, pRictor (T1135) and pAMPK (T172) were highlighted $(P<0.001)$. Meanwhile, the concordance indexes $(C$-indexes $)$ of the variables were summarized to investigate the discriminatory ability of random effects for overall survival status. Interestingly, the C-index of pAMPK (T172) (0.659) is much higher than that of pRictor (T1135) (0.591) and the other proteins (0.595-0.619) analyzed, which suggests AMPK as a prognostic predictor in our study group (Fig. 1a). Using the difference in expression of pAMPK (T172) between tumors and paired normal tissues as the cutoff (Fig. 1b), we also found that patients with higher relative expression of pAMPK (T172) in tumor tissues showed much lower overall survival rates than those with lower expression of pAMPK (T172) in tumor tissues (log-rank test, $P=0.009$, Fig. $1 \mathrm{~b}$ ). Furthermore, the hazard ratio of pAMPK (T172) was 2.376 (95\% CI: 1.359-4.152, $P=$ 0.0002 ), indicating that pAMPK (T172) is likely to play an independent role in determining the risks in stage II or III CRC (Table S3).

The RPPA analysis results suggest that AMPK $\alpha$ protein might be correlated with overall survival of stage II/ III CRC patients. Since phosphorylation of Thr172 in the AMPK $\alpha$ subunit is a critical event for its full activation, we thus assessed protein levels of AMPK $\alpha 1$, AMPK $\alpha 2$, and pAMPK (T172) and mRNA levels of AMPK $\alpha 1$ in different CRC cell lines. As expected, both the protein and mRNA levels of AMPK $\alpha 1$ were higher in almost all tumor cells compared with the levels in normal colon cell lines CCD112, CCD841, and NCM460 (Fig. S1A, B). In addition, we have also tested the mRNA expression of AMPK $\alpha 1$ and AMPK $\alpha 2$ in 24 CRC patients' tumor samples, in which AMPK $\alpha 1$ showed much higher expression than AMPK $\alpha 2$ in all tumor samples (Fig. 1c). In addition, protein levels of AMPK $\alpha 1$ and pAMPK (T172) were higher in most cancer samples compared with that in paired nontumorous tissues (Fig. 1d), which was further validated with immunohistochemistry assays (Fig. 1e).

Therefore, we further explored the prognostic performance of AMPK $\alpha 1$ in a larger cohort of CRC patients that 
A

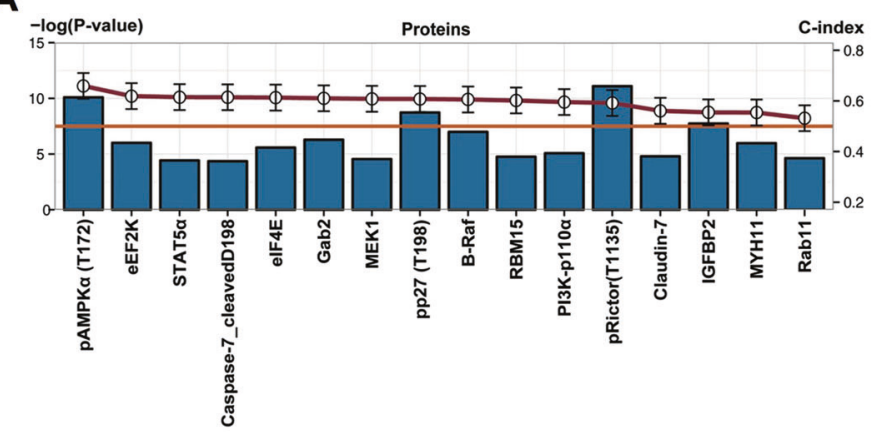

C

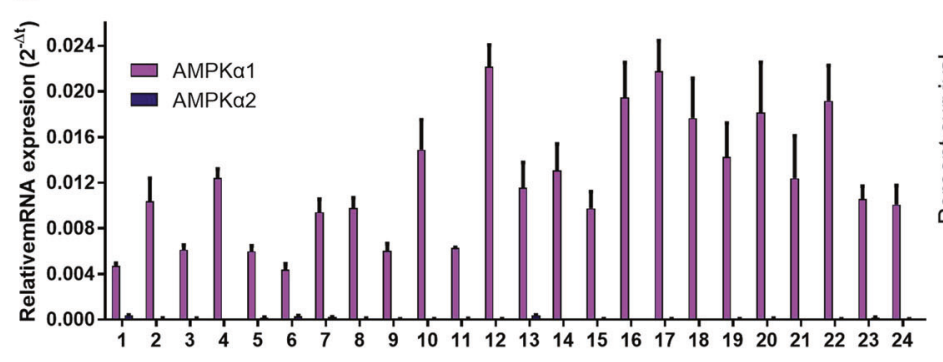

B
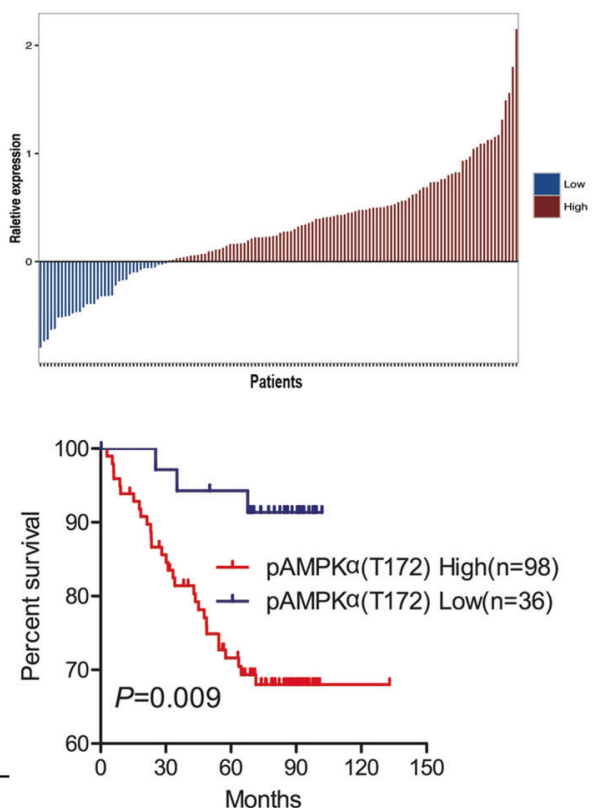

D

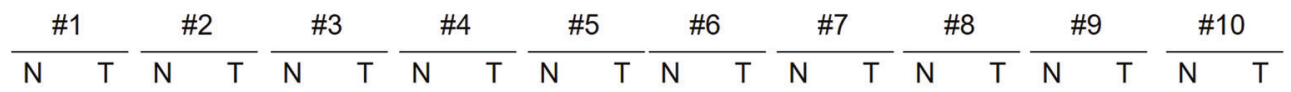

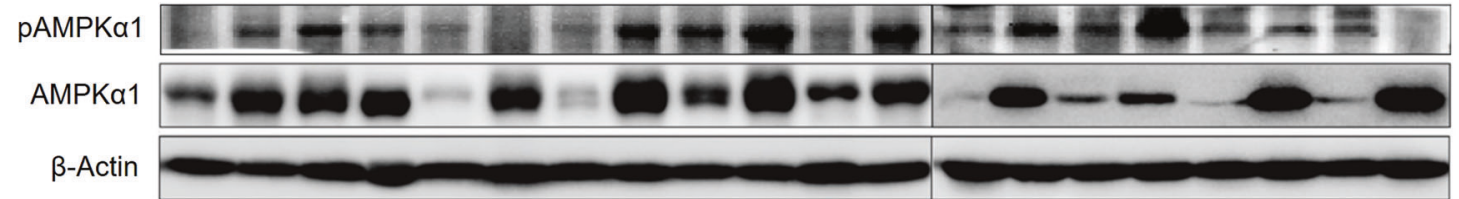

E

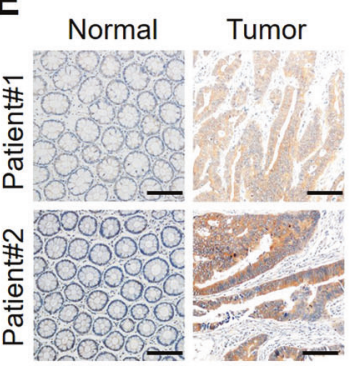

$\mathbf{F}$

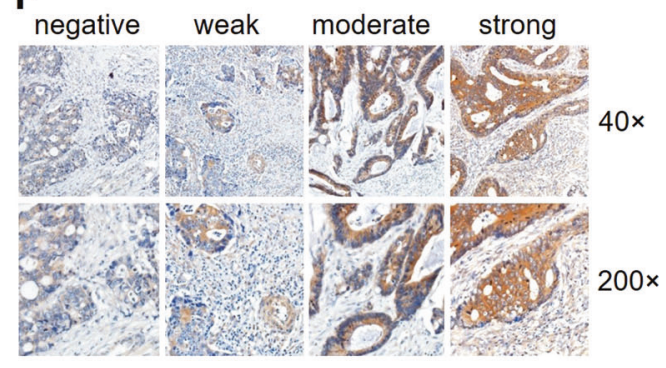

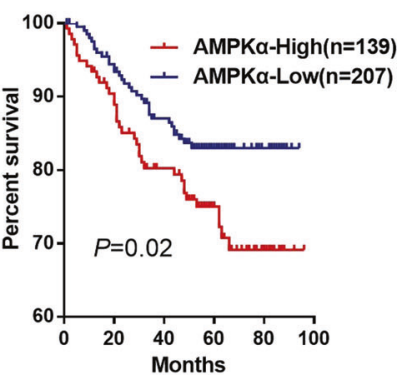

Fig. 1 Identification of AMPK $\alpha 1$ as prognostic predictor for stage II/III CRC patients. a 16 proteins were significantly correlated with overall CRC survival (univariate Cox regression analysis, Wald $P$ value $<0.05$, shown in bar plot). C-index values are also summarized in the line plot (red). pAMPK(T172) had the highest $\mathrm{C}$-index (0.659). Proteins from fresh cancer and normal tissues were detected using the RPPA platform. b Patients were assigned into high expression group and low expression group according to pAMPK (T172) expression (Upper panel). Kaplan-Meier (KM) survival curves of 134 CRC patients are stratified by expression levels of pAMPK(T172) (Log-rank test, $P=0.009$ ) (Lower panel). c mRNA

contained not only stage II/III but also stage I/IV. These 346 patients contained the previous 140 patients and comprised of 23 stage I, 144 stage II, 128 stage III, and 51 stage IV patients. Consistent with the RPPA findings, patients with higher expression levels of AMPK $\alpha 1$ showed expression of AMPK $\alpha 1$ was examined with qRT-PCR and indicated an upregulation in cancer tissues of 24 CRC patients compared with their nontumorous tissues. d Immunoblotting of AMPK $\alpha 1$ and pAMPK(T172) in ten paired nontumorous tissues and cancer tissues. e Immunohistochemistry of AMPK $\alpha 1$ in two representative paired nontumorous tissues and cancer tissues. Scale bar: $100 \mu \mathrm{m}$. f Left: Immunohistochemistry of AMPK $\alpha 1$ in patients with different stages of CRC cancer. Representative images are shown. Right: Kaplan-Meier (KM) survival curves of 346 CRC patients are stratified by $\mathrm{AMPK} \alpha 1$ immunohistochemistry scores

much lower overall survival rates than those with lower expression levels of AMPK $\alpha 1$ (Log-rank test, $P=0.02$, Fig. 1f). We concluded that the high levels of AMPK $\alpha 1$ were significantly correlated with poor overall survival in stage II/III CRC. 


\section{AMPKa1 is required for CRC cell survival and tumor growth}

To examine the function of AMPK $\alpha 1$ in CRC cells, we generated stable AMPK $\alpha 1$ knockdown RKO and HCT116 cells by using shRNA (Fig. 2a) and then challenged cells with glucose-free medium. Compared with their parental counterparts, cells expressing shRNA targeting AMPK $\alpha 1$ underwent greater cell death and impaired cell viability in RKO and HCT116 cells (Fig. 2b, c). In addition, similar results were observed in other two CRC cell lines SW1116 and DLD1 by using siRNA (Fig. S2). Consistent with these results, more apoptotic cells were detected in the knockdown group than in their counterparts (Fig. 2d). To determine tumorigenic effect of AMPK $\alpha 1$ in vivo, we injected the RKO and HCT116 cells with control or AMPK $\alpha 1$-shRNAs subcutaneously into nude mice and found that AMPK $\alpha 1$ knockdown significantly inhibited tumor growth (Fig. 2e, f). Taken together, these results demonstrate that AMPK $\alpha 1$ is required for CRC cell survival and tumor growth under conditions of energy stress in vitro and in vivo.

\section{AMPKa1 maintains cellular glutathione levels}

To determine how AMPK $\alpha 1$ affects cellular energy metabolism and regulates CRC cell survival, a metabolomic analysis was performed on HCT116 and RKO cell lines. Unsupervised principal components analysis revealed that the principal components of the metabolomic profiles obtained from the HILIC-ESIESI ${ }^{-}$-MS analysis did not show a separation of control NC-shRNA treated HCT116 cells from the AMPK $\alpha 1$-shRNA3-treated HCT116 cells, whereas the principal components in RKO cells did show an improved segregation (Fig. 3a). Supervised orthogonal partial least squares discriminant analysis (OPLS-DA) successfully discriminated between the NC-shRNA and AMPK $\alpha 1$-shRNA3-treated HCT116 or RKO cells (data not shown). Further analysis of the significant discriminatory metabolites was screened using variable importance in the projection scores (VIP $>1$ ) (Fig. 3b). The relative responses of glutathione $(P<0.01)$, glucose 1 -phosphate $(P<0.01)$, and uridine $(P<0.05)$ were significantly lower in AMPK $\alpha 1$ knockdown RKO cells (Fig. 3c). Moreover, the levels of glutathione were also lower in AMPK $\alpha 1$ knockdown HCT116 cells (Fig. 3c). Consistent with these results, AMPK $\alpha 1$ knockdown in RKO or HCT116 cells significantly decreased cellular glutathione levels (Fig. 3d).

\section{AMPKa1 maintains ROS at low levels through the regulation of GSR phosphorylation}

Glutathione is the most abundant cellular antioxidant, we then assessed cellular reactive oxygen species (ROS) levels.
AMPK $\alpha 1$ knockdown with siRNAs (Fig. 4a) dramatically increased total cellular ROS levels after glucose shortage (Fig. 4b). N-acetyl-cysteine (NAC), a precursor for glutathione synthesis, effectively suppressed cell death in AMPK $\alpha 1$ knockdown cells (Fig. 4c). Consistently, higher $\mathrm{NADP}^{+} / \mathrm{NADPH}$ ratios were observed in AMPK $\alpha 1$ knockdown CRC cells compared with the ratios in NC shRNA cells, which reflects NADPH depletion (Fig. 4d). Since AMPK $\alpha 1$ is a kinase and could phosphorylate enzymes, we proposed that glutathione synthesis might be regulated by AMPK $\alpha 1$-dependent phosphorylation. Addition of phosphor-tag into SDS-PAGE gels showed that several residues of glutathione synthesis reductase (GSR) were phosphorylated and the phosphorylation bands were weakened after silence of AMPK $\alpha 1$ (Fig. 4e). Consistently, treatment with Compound $\mathrm{C}$ or phenformin significantly suppressed or activated GSR phosphorylation in HCT116 cells (Fig. 4f).

To further confirm the phosphorylated residues of GSR, HPLC-tandem mass spectrometry (MS/MS) analysis was performed on tryptic digests of GSR immunoprecipitants from HCT116 transfected with lentivirus expressing NC or AMPK $\alpha 1$-shRNA after glucose deprivation. MS spectra showed residue Thr507, which is highly conserved among species, was found only in HCT116 NC-shRNA cells, but not found in AMPK $\alpha 1$-shRNA cells (Fig. 5a). According to the structure of GSR (Protein Data Bank ID: 3dk8), Thr507 lies adjacent to His511, proton acceptor of GSR, whereby phosphorylation might play critical roles in its activity (Fig. 5b). We next produced specific antibody recognizing phosphorylation of Thr507 in GSR (pGSR). Figure $5 \mathrm{c}$ demonstrated that glucose deprivation induced Thr507 phosphorylation of GSR was AMPK $\alpha 1$-dependent, while total GSR levels were not affected by AMPK $\alpha 1$ expression. To further determine phosphorylation of Thr507 in GSR was involved in glucose stress resistance, we mutated Thr507 into the phosphorylationimitated Asp507 (T507E) and Glu507 (T507D) and negative control Val507 (T507V) and then transfected them in AMPK $\alpha 1$ and GSR double knockdown HCT116 and RKO cells (Fig. S3). Enzymic activity assays revealed that compared with cells expressing the GSR-WT, GSR activity was decreased in cells transfected with the T507V mutation vector and enhanced in cells transfected with the T507E and T507D mutation vector (Fig. 5d). To test the ability of the GSR Thr507 phosphorylation to support the survival of HCT116 and RKO cells in AMPK $\alpha 1$ knockdown cells upon glucose shortage, we performed flow assays to detect the apoptotic proportion in these cells. The results showed that GSR T507D mutant partly reversed CRC cell death caused by AMPK $\alpha 1$ and GSR double knockdown (Fig. 5e). In the clinical samples, immunestaining with pGSR correlated positively with AMPK $\alpha 1$ 


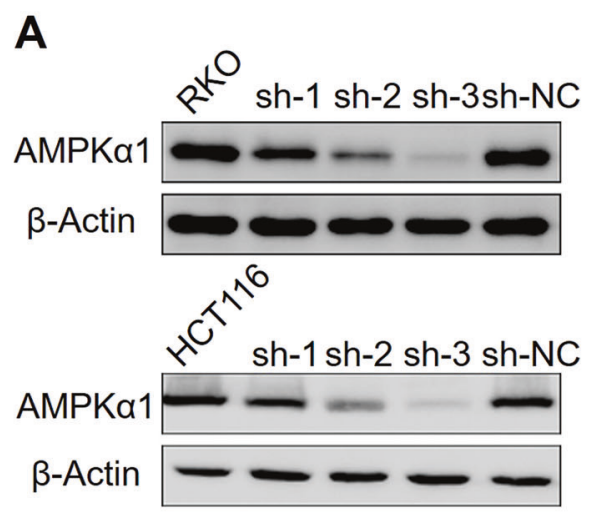

B
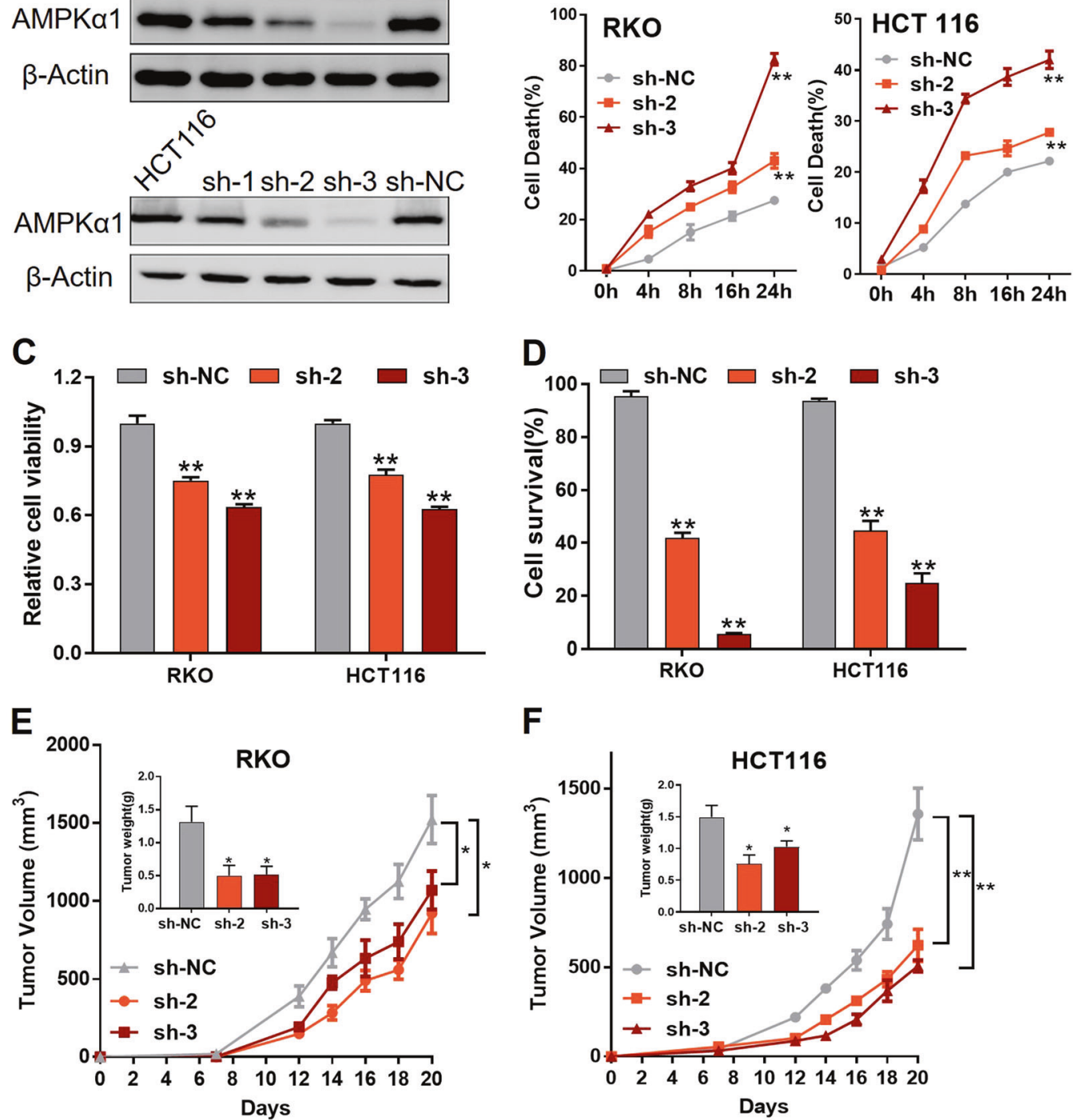

$\mathbf{F}$
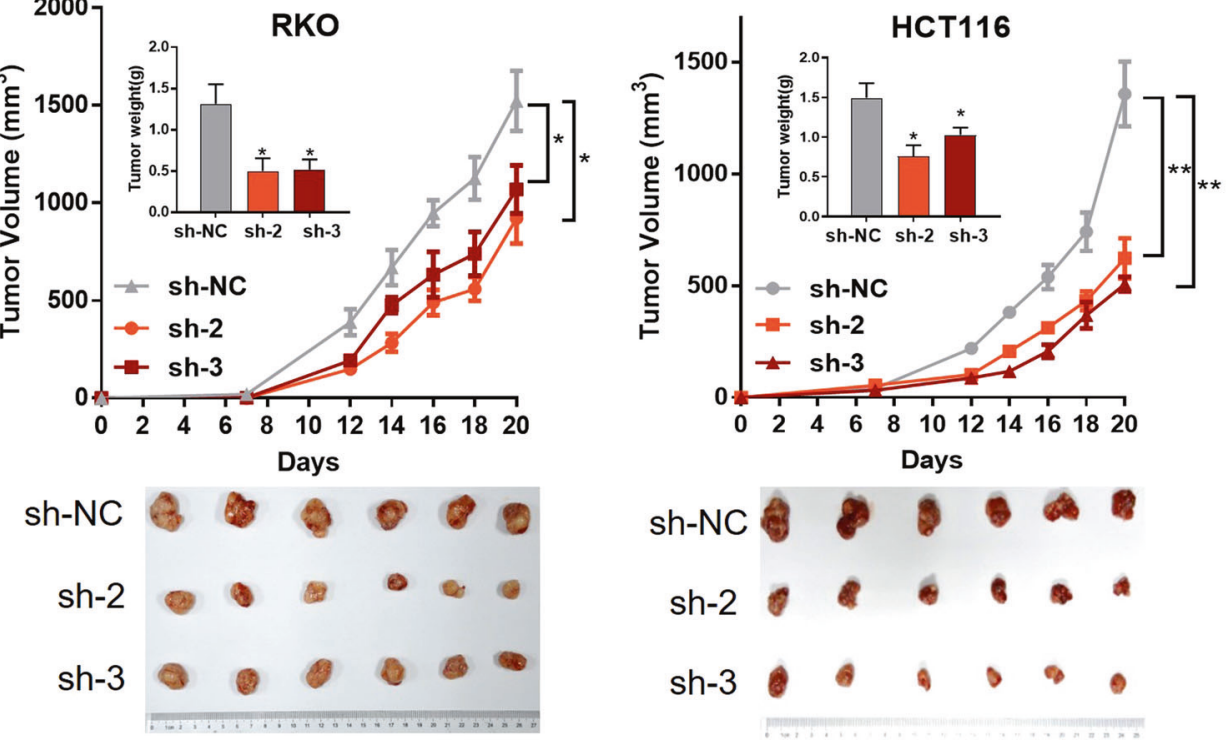

Fig. 2 Expression of AMPK $\alpha 1$ maintains tumor cell survival in the absence of glucose. a Immunoblotting of AMPK $\alpha 1$ transfected with lentiviruses expressing different shRNAs in RKO and HCT116 showed that sh-3 had the highest efficiency and sh-2 the median. b RKO and HCT116 cells expressing control-shRNA (sh-NC), sh-2 RNA, or sh-3 RNA are cultured in glucose-free medium for the indicated time points to quantify cell death via a trypan exclusion assay. $\mathbf{c}$ Viability of RKO and HCT116 cells was detected by CCK- 8 assay in the absence of

glucose for $24 \mathrm{~h}$. d Apoptosis of RKO and HCT116 cells cultured in the absence of glucose for $24 \mathrm{~h}$ was detected by flow cytometry. RKO (e) and HCT116 (f) cells expressing control-shRNA (sh-NC), sh-2 RNA, or sh-3 RNA were subcutaneously injected into the right armpit of nude mice and tumor volumes were recorded three times per week. Tumor tissues dissected from each group were photographed. $* P<$ $0.05, * * P<0.01$, Student's $t$-test

expression (Fig. 5f). Collectively, these results indicated that GSR Thr507 is most likely the inducible phosphorylation substrates of AMPK $\alpha 1$ in CRC cells under glucose

deprivation and that it plays critical roles in GSR activity and likely functions accounting for the protective roles of AMPK $\alpha 1$ in cell survival under nutrient stress. 
A

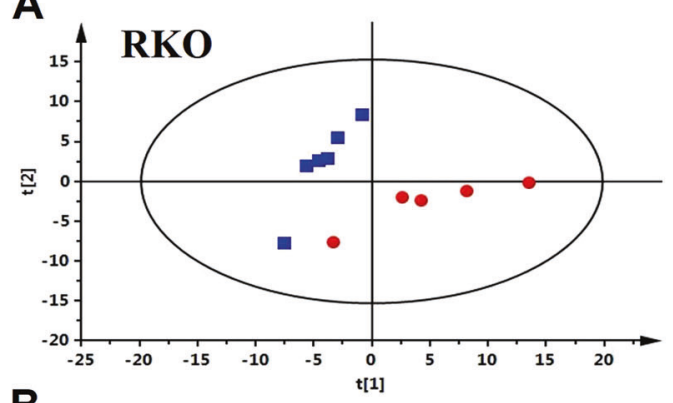

B

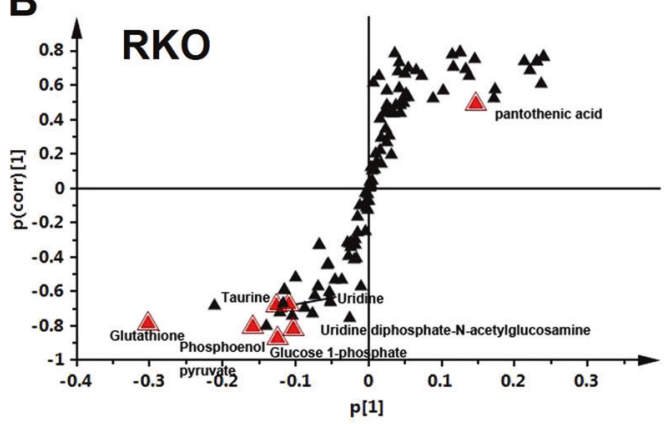

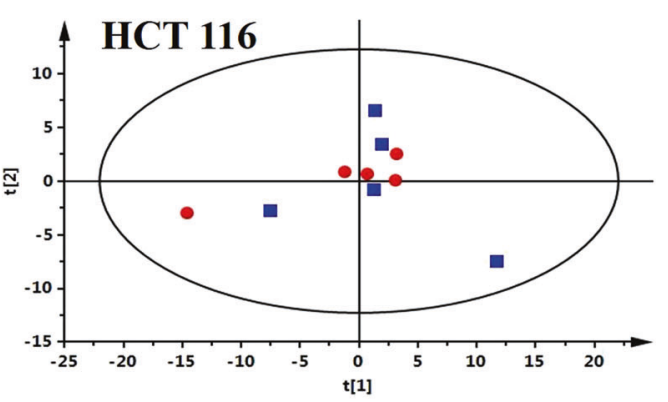

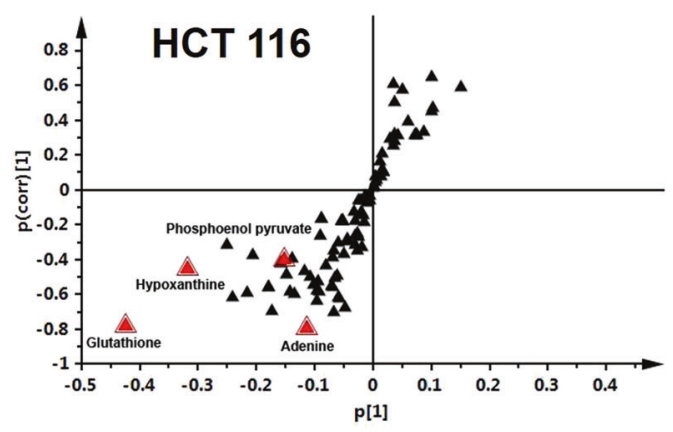

C

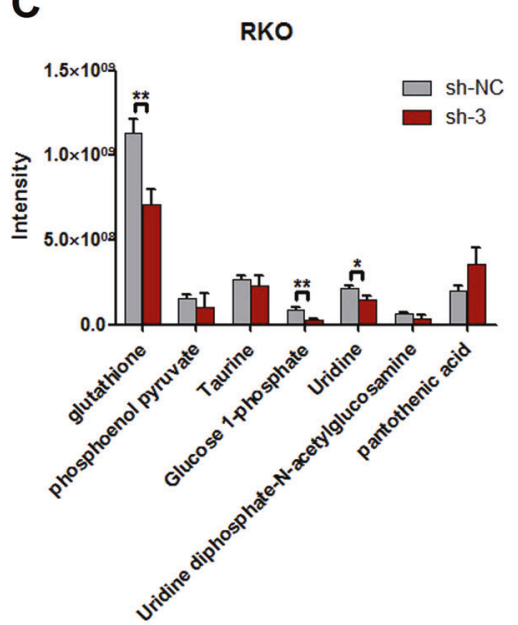

D

RKO
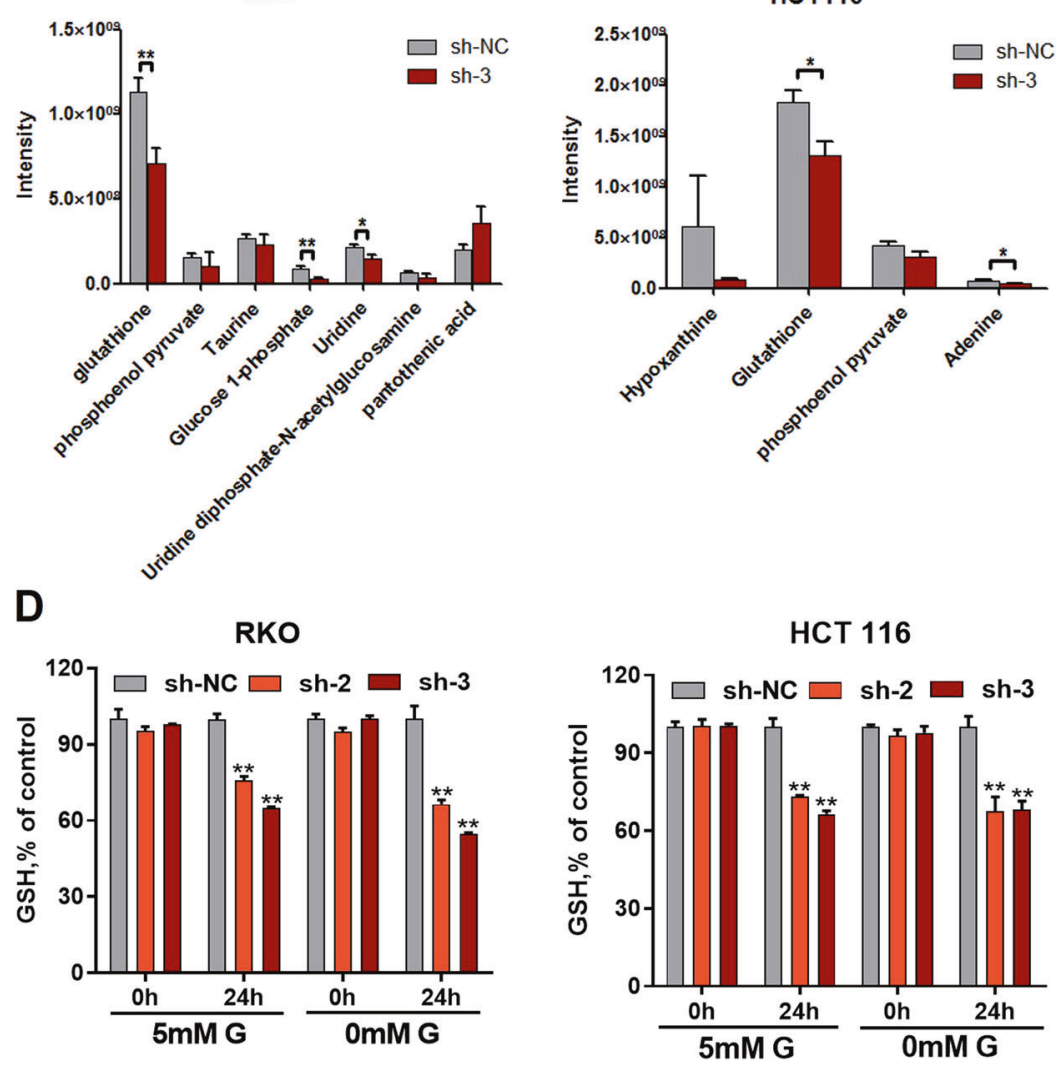

Fig. 3 Expression of AMPK $\alpha 1$ maintains cellular glutathione levels. a Scatter plot of scores from the PCA analysis of RKO and HCT116 cells expressing control-shRNA (sh-NC, blue boxes) or \#3-shRNA (sh-3, red dots) under the negative ionization mode. b S-plot of OPLSDA analysis of RKO and HCT116 cells expressing control-shRNA (sh-NC) or \#3-shRNA (sh-3). c Relative cellular abundance of

metabolites was different in RKO and HCT116 cells expressing control-shRNA (sh-NC) or \#3-shRNA (sh-3). d Identification of cellular GSH depletion in RKO and HCT116 cells expressing controlshRNA (sh-NC) or \#3-shRNA (sh-3) incubated in $5 \mathrm{mM}$ glucose or glucose-free medium. Data are presented as the mean $\pm \mathrm{SD}(n=6)$. $* P<0.05,{ }^{*} * P<0.01$, Student's $t$-test 

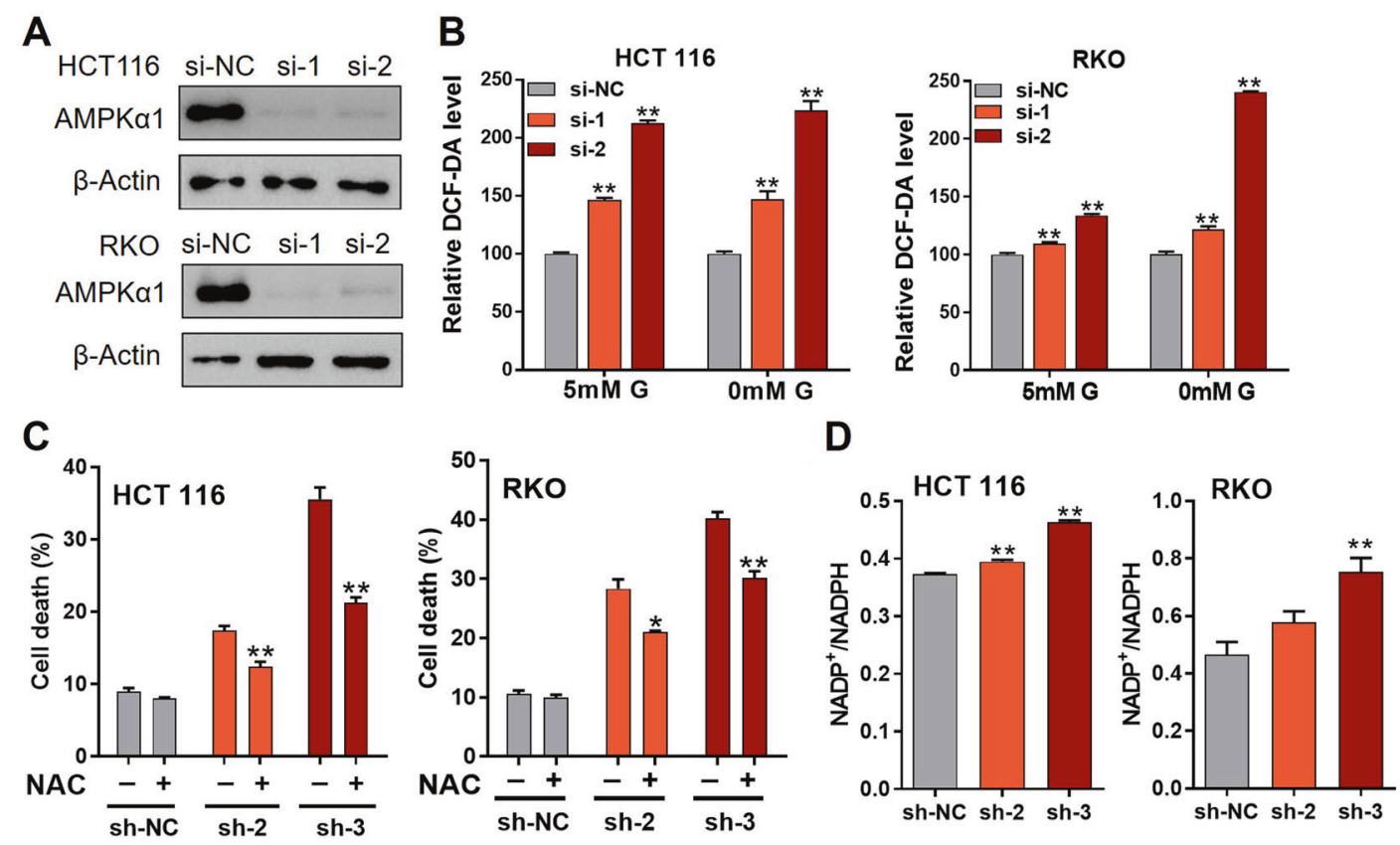

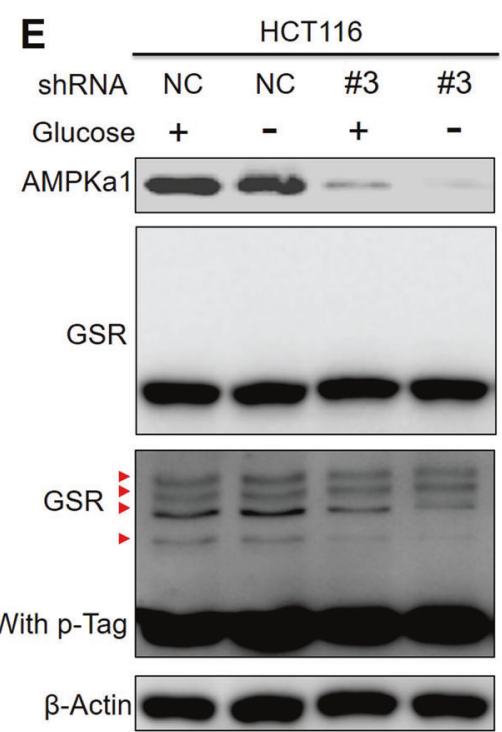

Fig. 4 Phosphorylation of GSR by AMPK $\alpha 1$ was responsible for ROS maintenance. a Knockdown efficiency of siRNA targeting AMPK $\alpha 1$ was validated by western blot analysis. b RKO and HCT116 cells were transfecting with siRNA targeting AMPK $\alpha 1$ before incubation with $5 \mathrm{mM}$ glucose or glucose-free medium and the cellular ROS production was detected with DCF-DA staining. $\mathbf{c}$ RKO and HCT116 cells expressing control-shRNA (sh-NC), sh-2 RNA, or sh-3 RNA were cultured in glucose-free medium with or without NAC $(3 \mathrm{mM})$ for $18 \mathrm{~h}$ before cell death was quantified by trypan exclusion staining. d RKO and HCT116 cells expressing sh-NC, sh-2 RNA, or sh-3 RNA were cultured in glucose-free medium for $12 \mathrm{~h}$ before measurement of the intracellular $\mathrm{NADP}^{+} / \mathrm{NADPH}$ ratio. e Total cell lysates from RKO and HCT116 cells cultured with or without glucose were subjected to Phos-tag gel electrophoresis and immunoblotted for GSR. pGSR: red triangle. Top shows immunoblot for GSR without Phos-tag. f Total cell lysates from HCT116 cells treated with Compound C or phenformin were subjected to phos-tag gel electrophoresis and immunoblotted for GSR. $* P<0.05, * * P<0.01$, Student's $t$-test

\section{AMPKa1 is a potential target in CRC}

Chemotherapeutic agents were previously reported to induce redox stress in cancer cells [20]. We therefore hypothesized that knockdown of AMPK $\alpha 1$ could sensitize CRC cells to oxaliplatin treatment. Flow assays showed increased cell apoptosis after silence of AMPK $\alpha 1$ when treated with oxaliplatin in HCT116 and RKO cells (Fig. 6a). Treatment with oxaliplatin induced significant elevated ROS levels in AMPK $\alpha 1$ knockdown cells (Fig. 6b). In addition, we detected the IC50 of oxaliplatin in 10 CRC cell lines (Table S4). We found HCT116 and RKO cell lines are relatively sensitive to oxaliplatin. We also evaluated the effect of AMPK $\alpha 1$ knockdown on oxaliplatin sensitivity in 
A

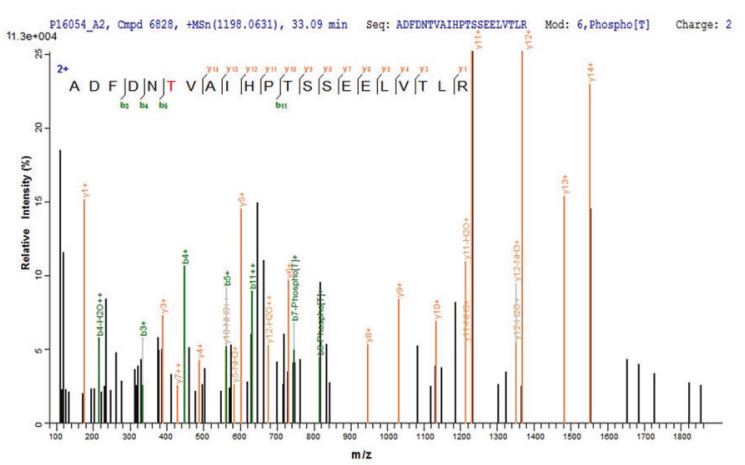

ADFDNTVAIHPTSSEELVTL Tandem MS:T507 ATKADFDNTVAIHPTSSEELVTL Homo sapiens ATKADFDNTVAIHPTSSEELVTL Rattus norvegicus ATKADFDNTVAIHPTSSEELVTL Mus musculus ATKADFDNTVAIHPTSSEELVTL Macaca mulatta
B

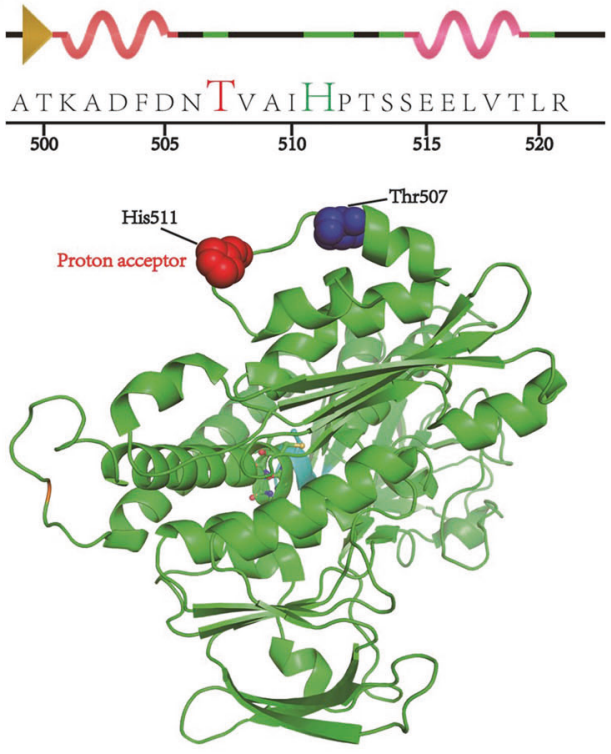

C

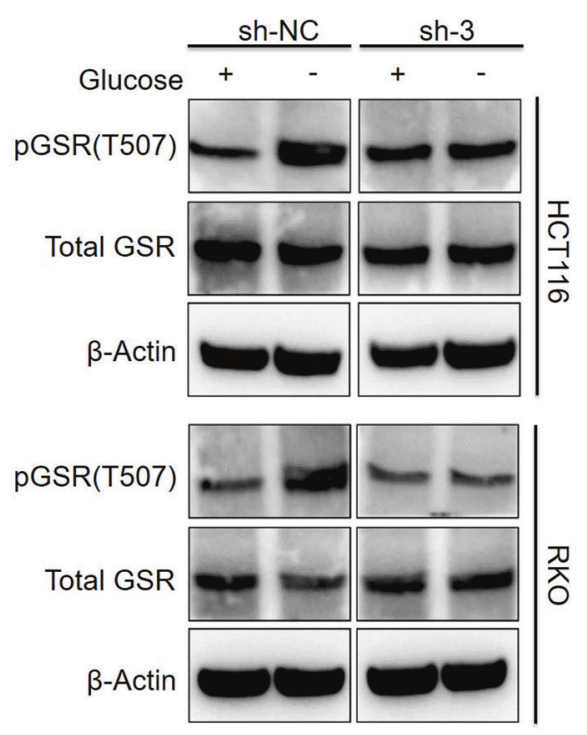

F

Patient\#1

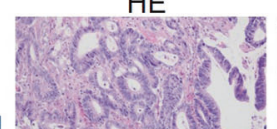

AMPKa1

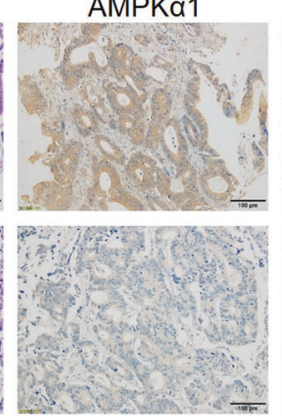

D

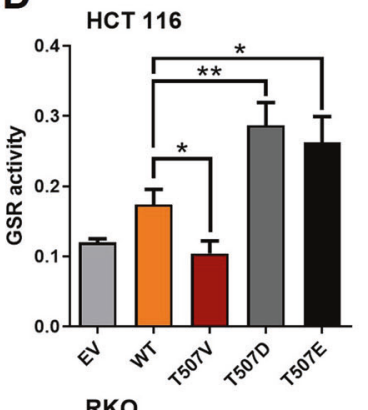

E
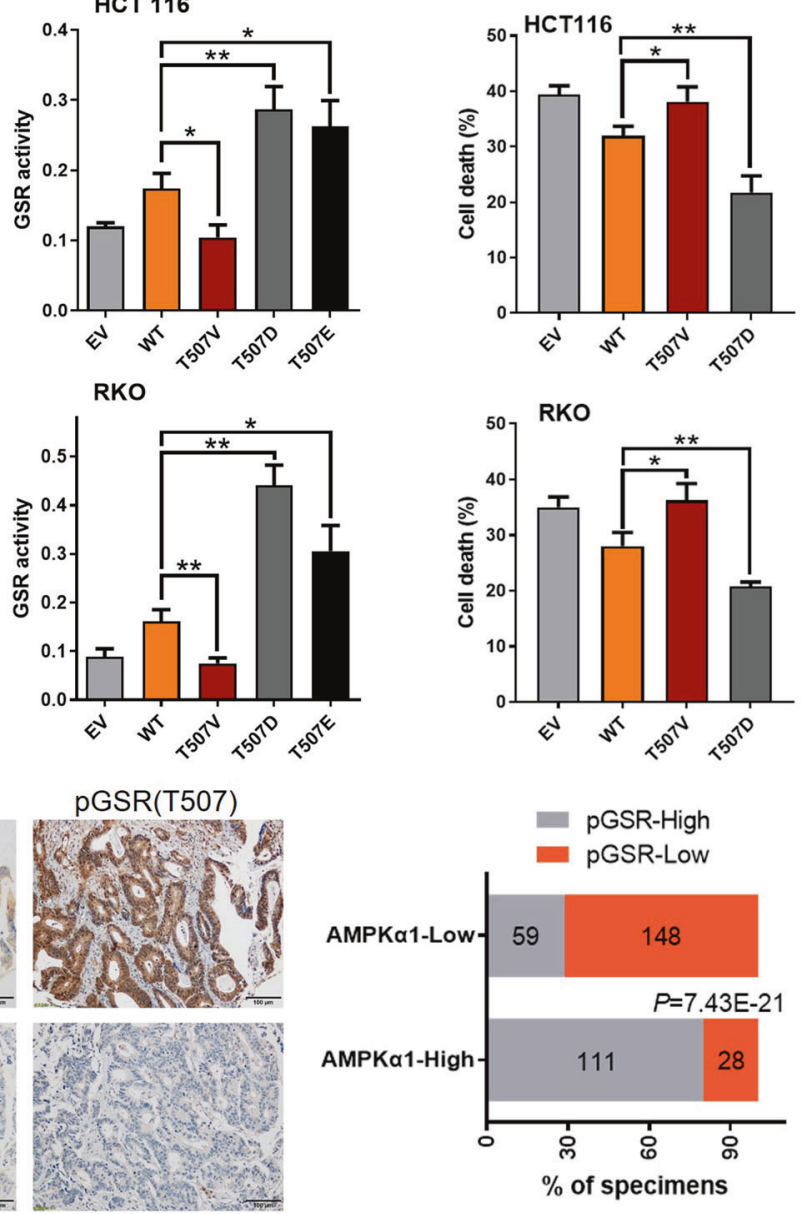

oxaliplatin-resistant SW1116 and DLD1 cells. The results showed that this effect was not cell line dependent (Fig. 6c). In order to confirm the in vitro results, we constructed a
shRNA delivery system, HA-quaternary polyplex, to validate the therapeutic effects of AMPK $\alpha 1$ knockdown in vivo. The characteristics and function of the polyplex was 
Fig. 5 Thr507 phosphorylation of GSR is important for the survival function of AMPK $\alpha 1$. a MS/MS spectra of phosphopeptides containing the phosphotyrosine (pThr) 507 site of GSR. Fragment ions are shown, as is the sequence coverage due to identified fragment ions. All of the highest peaks were explained, but for clarity, they are not all annotated. $\mathrm{m} / \mathrm{z}$, mass-to-charge ratio (Upper panel). Thr507 phosphorylation site is highly conserved among human, rat, mouse, and monkey. Identified phosphopeptide are shown above the sequence alignments (Lower panel). b The identified peptide containing the phosphorylated site was shown in two dimension. The phosphorylated site Thr507 and the proton acceptor His511 were indicated in red and green, respectively (Upper panel). Thr507 in the crystal structure of human GSR. Overall view of GSR (Protein Data Bank ID code 3dk8) was shown. The relative positions of His511 (red spheres) and Thr507 (cyan spheres) are indicated (Lower panel). c Immunoblotting of pGSR(T507), total GSR in RKO, and HCT116 cells cultured in glucose deprivation conditions. d GSR enzymatic activities of T/V, T/D, and T/E mutations of Thr507 in HCT116 and RKO cells. e Cell apoptosis of T/V and T/D upon glucose shortage was determined by annexin V/PI staining. f Left: Immunohistochemistry of AMPK $\alpha 1$ and pGSR(T507) in CRC clinical samples. Representative images including corresponding hematoxylin and eosin staining are shown. Right: Scoring of pGSR(T507) in AMPK $\alpha 1$ low and high samples and correlation analysis by using Chi-square test. Results are representative of three experiments. $* P<0.05, * * P<0.01$, Student's $t$-test, Chisquare test

confirmed in Fig. S4. The AMPK $\alpha 1$-shRNA3 treatment groups result in significant inhibition of tumor growth compared with the control group (Fig. 6d, e and S5A). Moreover, the body weight and histological analysis of organs of mice treated with in PPMS polyplexes did not differ from that of the control, indicating that the PPMS polyplexes did not exhibit severe systemic toxicity (Fig. S5B, C). In addition, the specific downregulations of AMPK $\alpha 1$ on both mRNA and protein levels were observed in the PPMS polyplexes/AMPK $\alpha 1$-shRNA3 treated mice group (Fig. S5D, E). Besides PPMS polyplexes, AMPK inhibitor Compound $\mathrm{C}$ and oxaliplatin were used for combination therapy in mouse model. The results showed that Compound $\mathrm{C}$ could enhance the sensitivity of CRC tumors to oxaliplatin treatment (Fig. S6).

To further explore the relationship between AMPK $\alpha 1$ expression and oxaliplatin efficacy in clinical data, IHC staining for AMPK $\alpha 1$ was performed on tumor tissues from 76 patients with advanced CRC treated with FOLFOX or XELOX regimens as first line therapy. Overall 27 of 32 $(84.3 \%)$ patients with low AMPK $\alpha 1$ expression in their primary tumors benefited $(\mathrm{CR}+\mathrm{PR}+\mathrm{SD})$ from chemotherapy, whereas only 18 of 44 (40.9\%) patients with high AMPK $\alpha 1$ expression benefited from the therapy (Fig. S7A), suggesting that low AMPK $\alpha 1$ expression predicts a favorable response to oxaliplatin-based chemotherapy. Patients with response to oxaliplatin-based chemotherapy tends to showed lowered AMPK $\alpha 1$ expression than those resistant to oxaliplatin (Fig. S7B). Strikingly, the progression-free survival of patients with low $\mathrm{AMPK} \alpha 1$ protein expression was dramatically longer than that of patients with high AMPK $\alpha 1$ expression $(P<0.05$; Fig. S7C). We then generated three patient-derived xenografts to evaluate therapeutic potential of AMPK $\alpha 1$ silence. As shown in Figs. 6f and S8, combination of HA-quaternary/AMPK $\alpha 1$-shRNA3 and oxaliplatin significantly suppressed tumor growth compared with oxaliplatin alone in the mice.

On the basis of the above observations, we concluded that downregulating the expression of AMPK $\alpha 1$ could significantly inhibit tumor growth in subcutaneous cell-linederived xenograft model and patient-derived xenograft model, which highly suggests the potential of AMPK $\alpha 1$ gene silencing as a therapeutic target for CRC treatment.

\section{Discussion}

Maintaining metabolic homeostasis under conditions of energy stress is essential for cancer cell survival and induces cancer recurrence and metastasis following radical treatments. Our study aimed to address this important clinical issue and found that AMPK $\alpha 1$ may be a predictor for the prognosis of CRC patients. Mechanistic studies show that AMPK $\alpha 1$ is critical for CRC cell survival, and deletion of AMPK $\alpha 1$ renders cancer cells susceptible to physiological damage by ROS via alterations in GSR phosphorylation and a subsequent decrease in reduced glutathione. In some genetic analyses, AMPK has been shown to behave as a tumor suppressor and a number of drugs that activate AMPK also suppress cell growth [21, 22]. However, several studies have indicated that physiological AMPK activation is pro-tumorigenic [18]. Previous studies have not been performed on samples from patients that represent the in vivo physiological microenvironment. Our results are consistent with the findings from studies on myeloid leukemia in which AMPK was shown to protect leukemia-initiating cells from metabolic stress in bone marrow [19]. CRC shows an adaptive response to hypoxia via an upregulation of anaerobic glycolysis and angiogenesis [23]. Our results demonstrate that AMPK $\alpha 1$ can aide in the survival of CRC cells under physiological metabolic stress, which may lead to disease recurrence as well as a poor prognosis.

Our results further support the notion that AMPK $\alpha 1$ and AMPK $\alpha 2$ may have different functions. These two isoforms of AMPK $\alpha$ have shown specific effects in normal tissues and may also perform different functions in cancer cells $[17,24,25]$. In humans, AMPK $\alpha 1$ is ubiquitously distributed while AMPK $\alpha 2$ is abundant in skeletal and cardiac muscle [26]. We observed uniformly higher expression levels of AMPK $\alpha 1$ in patients' samples and CRC cell lines than in nontumorous samples and normal cell lines; while the expression levels of AMPK $\alpha 2$ were 
quite low in patients' samples. Higher expression of AMPK $\alpha 1$ in tumors than in normal tissue was also observed in other cancer types such as pancreatic and cervical cancer $[27,28]$. Silencing of AMPK $\alpha 1$ with RNA interference inhibited the growth of pancreatic cancer cells in vitro and in vivo [28]. In chondrosarcoma cells and pancreatic cancer cells, the inhibition of both AMPK $\alpha 1$ and AMPK $\alpha 2$ reduced cell migration and tumor growth $[26,29]$. The role of AMPK $\alpha 1$ may vary due to the different cell types in which the cancer was initiated. Our results suggest that different roles might be played by AMPK $\alpha 1$ and AMPK $\alpha 2$ in CRC. Loss of AMPK $\alpha 1$ expression has also been associated with poor survival in melanoma patients, which suggests AMPK $\alpha 1$ may perform different roles in different tumor types [30].

Cancer cells frequently exhibit high levels of oxidative stress and an upregulation in their antioxidant capacity [31]. On one hand, the elevation of ROS in cancer promotes cell proliferation, cell survival, and tumor development [32]. On the other hand, when the increase in ROS reaches a certain level, it may overwhelm the antioxidant capacity of the cells and trigger cell death [31]. Glutathione is the most abundant antioxidant in cells and acts to maintain redox balance [33]. Compounds which target the glutathione antioxidant system and cause severe ROS accumulation have been shown to preferentially kill cancer cells and prolong animal survival [34-36]. Current phosphorylation profiling has identified several phosphorylation sites in GSR [37, 38]. However, the function and regulatory mechanism of these sites remain unknown. Our study, for the first time, identified AMPK $\alpha 1$ as an upstream regulator of GSR possibly through phosphorylation at residue Thr507 and thus increase its activity.

To evaluate the therapeutic effect on AMPK $\alpha 1$, we specifically developed a modified PPMS/shRNA polyplexes for efficient systemic gene delivery to manipulate the gene expression levels of AMPK $\alpha 1$ in CRC models. Our results clearly demonstrated that the HA-quaternary polyplexes could effectively deliver AMPK $\alpha 1$ shRNA to CRC cells in vivo and that downregulation of the expression of AMPK $\alpha 1$ could significantly inhibit tumor growth in subcutaneous CRC models. We also found AMPK $\alpha 1$ inhibition is synergistic with oxaliplatin both in cell-line-derived xenograft model and patient-derived xenograft model. This study proved that AMPK $\alpha 1$ could be potentially used as a therapeutic target for CRC treatment. But it still need further study to investigate the optimal way of AMPK $\alpha 1$ inhibition in humans.

In conclusion, our study demonstrates that the upregulation of AMPK $\alpha 1$ in CRC promotes cancer cell survival under conditions of energy stress and leads to a poor prognosis through maintaining cellular glutathione by regulating glutathione reductase phosphorylation. The strategy of AMPK $\alpha 1$ inhibition may be applied to treat CRC patients.

\section{Materials and methods}

\section{Patients, samples, and immunohistochemical analysis}

Fresh tissue samples from 140 patients with stage II - III CRC from Sun Yat-sen University Cancer Center were stored in liquid nitrogen immediately after surgery until used for analysis. All samples were authenticated by hematoxylin and eosin (H\&E) staining and those with tumor cells more than $40 \%$ were subjected to protein extraction using radioimmunoprecipitation assay (RIPA) buffer (Cell Signaling Technology, MA, USA). A functional proteomic analysis of these samples was conducted via RPPA analysis. Meanwhile, formalin-fixed paraffinembedded tissues of a cohort containing 346 patients were used for the immunohistochemical analysis of AMPK $\alpha 1$ according to previously reported protocols [39]. The tumor tissues of 76 patients with advanced CRC treated with FOLFOX or XELOX regimens were collected from Sun Yat-sen University Cancer Center. The AJCC criteria were used to classify the clinical and clinic-pathological stages and patient consent and approval was obtained from the Institutional Research Ethics Committee.

To quantify AMPK $\alpha 1$ or pGSR(T507) protein expression, both the intensity and extent of immunoreactivity were evaluated and scored. In the present study, IHC intensity was scored as follows: 0, negative staining; 1, weak staining; 2 , moderate staining; 3 , strong staining. The scores of the extent of immunoreactivity ranged from 0 to 3 and were according to the percentage of cells that had positive staining in each microscopic field of view $(0,<25 \% ; 1$, $25-50 \% ; 2,50-75 \% ; 3,75-100 \%)$. A final score ranging from 0 to 9 was achieved by multiplying the scores for intensity and extent. AMPK $\alpha 1$ or pGSR(T507) expression levels were considered high when the final scores were $\geq 4$ and low when the final scores were $<4$.

\section{Cell lines and cell culture}

Human CRC cell lines SW480, HCT116, SW1116, SW620, HCT8, HT-29, DLD-1, RKO, Ls174T, and LoVo and the colon epithelial cell line CCD112, CCD841 were purchased from the American Type Culture Collection (Manassas, VA, USA) in 2009 and cultured according to the instructions with 10\% FBS (Thermo Fisher Scientific, CA, USA) supplementation. All cells were cultured at $37^{\circ} \mathrm{C}$ with $5 \%$ $\mathrm{CO}_{2}$. All the above cells were authenticated by short tandem repeat DNA fingerprinting and tested for mycoplasma 
A
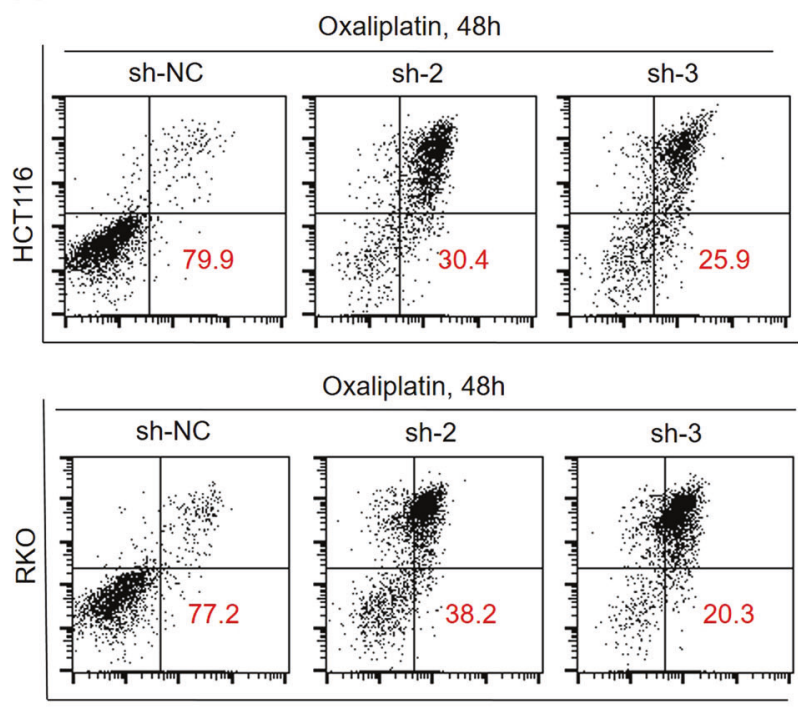

C

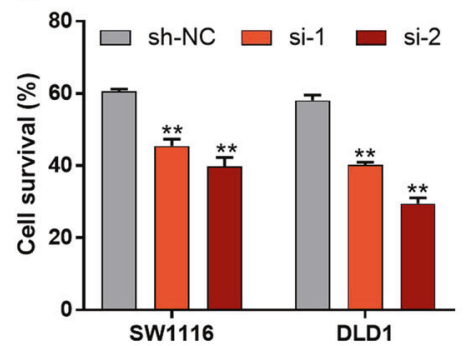

$\mathbf{F}$

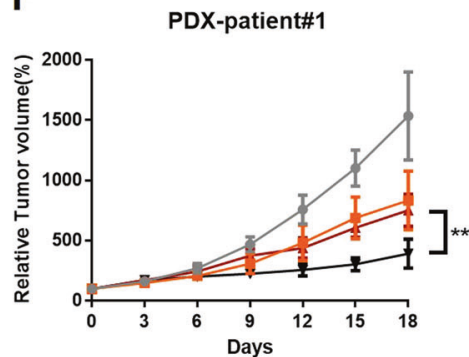

D

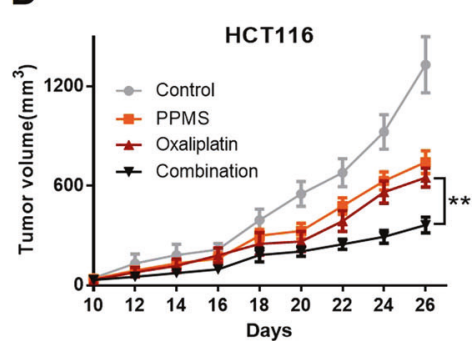

B

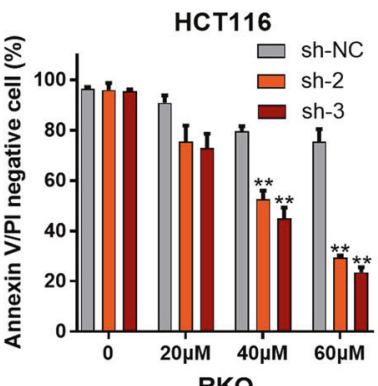

RKO

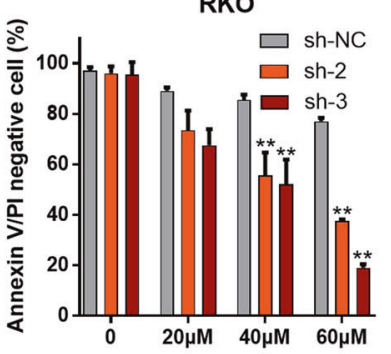

E
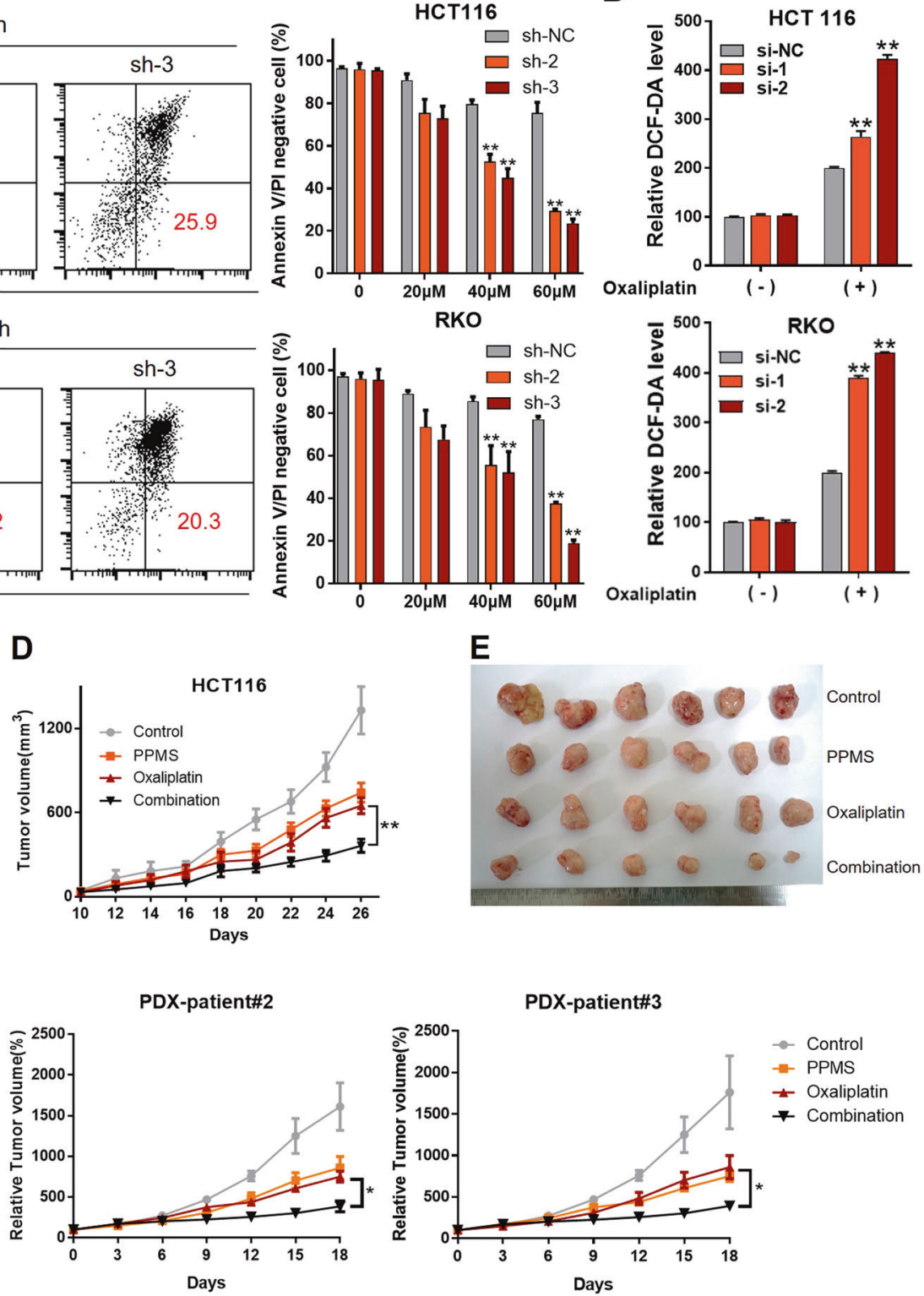

Fig. 6 Inhibition of AMPK $\alpha 1$ is synergistic with oxaliplatin. a Cell apoptosis in HCT116 and RKO cells expressing control-shRNA (shNC), sh-2 RNA, or sh-3 RNA after oxaliplatin treatment. Representative images and quantification are shown. b Intracellular ROS levels in HCT116 and RKO cells were detected with DCF-DA. c Cell survival of SW1116 and DLD1 cells treated by oxaliplatin $(60 \mu \mathrm{M})$

was measured by CCK-8 assay. d Tumor volumes after tail vein administration of different formulas until day 26. e The images of HCT116 tumor tissue after indicated treatments. f Tumor volumes after tail vein administration of different formulas until day 18 in a PDX model. $* P<0.05, * * P<0.01$, Student's $t$-test

before using. Glucose-free media were obtained from Life Technology (CA, USA).

\section{Reagents and antibodies}

Purified D-glucose and DCF-DA were obtained from Life Technologies. Oxaliplatin and NAC were purchased from Selleck Chemicals (TX, USA). Antibodies used for immunoblotting included those for AMPK $\alpha 1$ (Millipore, MA, USA, MABS818), pAMPK(Thr172) (Cell Signaling
Technology, MA, USA, \#2535), AMPK $\alpha 2$ (CST, \#2725), GSR (Abcam, Cambridge, MA, USA, ab16801), vinculin (Abcam, ab129002), GAPDH (ab128915), and $\beta$-actin (CST, \#4970).

\section{Real-time PCR}

Total RNAs were obtained with TRIzol reagent (Life Technologies, CA, USA) and reverse transcribed to cDNA using a PrimeScript RT Master Mix kit (Takara, NHK, 
Japan). mRNA expression levels were detected using realtime PCR according to a previous report [39]. Synthesized primers from Life Technologies were as follows:

AMPK $\alpha 1$-Forward: GGCACGCCATACCCTTGAT AMPK $\alpha 1-$ Reverse: TCTTCCTTCGTACACGCAAATAA AMPK $\alpha 2$-Forward: GTGAAGATCGGACACTACGTG AMPK $\alpha 2$-Reverse: CTGCCACTTTATGGCCTGTTA GAPDH-Forward: ATCACCATCTTCCAGGAGCGA GAPDH-Reverse: CCTTCTCCATGGTGGTGAAGAC

\section{Western blot}

Proteins were extracted with RIPA buffer and quantized using a BCA assay (Thermo Fisher Scientific, CA, USA). A total of $40 \mu \mathrm{g}$ were loaded and analyzed by western blotting as previously described $[39,40]$.

\section{Cell apoptosis, cell death, and proliferation assays}

Cell apoptosis induced by culturing with glucose-free medium was determined by annexin V/PI staining (4A Biotech Co, Beijing, China) followed by flow cytometry, according to the manufacturer's instructions. A trypan blue exclusion assay was conducted to detect cell death after exposure to glucose-free medium at the indicated time points. CCK-8 and colonic assay were used to detect cell viability and proliferation as described previously [41, 42].

\section{Plasmid}

The opening read frame of GSR (NM_000637) was cloned to the plasmids of pCMV6-Flag-empty (Genecopoeia ${ }^{\mathrm{TM}}$, MA, USA) and denominated as (GSR-WT). The targeted point mutation of GSR including T507V, T507D, and T507E was generated with the MutanBEST kit (Takara, NHK, Japan) according to the manufacturer's instructions.

\section{Immunoprecipitation}

Cells were rinsed twice with ice-cold PBS and lysed in icecold lysis buffer $(50 \mathrm{mM}$ Tris [pH 8.0], $150 \mathrm{mM} \mathrm{NaCl}$, $5 \mathrm{mM}$ EDTA, $0.1 \% \mathrm{NP} 40$ ) and $1 \%$ of protease inhibitors (Selleck, TX, USA). The cell lysates were isolated by centrifugation at $13,000 \mathrm{rpm}$ for $15 \mathrm{~min}$. For immunoprecipitations, the Flag-beads (Sigma-Aldrich, MO, USA) were co-incubated with the soluble supernatant in $4{ }^{\circ} \mathrm{C}$ overnight. Immunoprecipitates were washed three times with lysis buffer. Cell extracts or immunoprecipitated proteins were denatured by the addition of loading buffer followed by boiling for $5 \mathrm{~min}$, resolved by $10 \%$ SDSPAGE, and then transferred to nitrocellulose-ECL membranes (Millipore, Darmstadt, German) and incubated with antibodies against phospho-(Ser/Thr) (Abcam, MA, USA).
The immune complex was detected by chemiluminescence (Thermo Fisher Scientific, CA, USA).

\section{Enzyme assays}

The enzymatic activities of GSR was measured with the Glutathione Reductase Activity Colorimetric Assay Kit (Biovision, CA, USA) and conducted according to the manufacturer's instructions. Briefly, the cells transfected with the indicated vectors were homogenized on ice in four volumes of cold assay buffer and the supernatants were collected for assay after centrifugation at $10,000 \times g$ for $15 \mathrm{~min}$. The samples were mixed with catalase, incubated with reaction buffer after depletion of endogenic glutathione. The colorimetric intensity of samples was detected on a Synergy ${ }^{\mathrm{TM}}$ Multi-Mode Microplate Reader (Biotek, VT, USA) at a wavelength of $405 \mathrm{~nm}$. The GSR activity in the samples was calculated according to the standard curve.

\section{In vivo tumor growth}

Female athymic BALB/c nude mice (4-5 weeks of age, 15-18g) were purchased from Guangdong Province Laboratory Animal Center. All animal studies were performed in accordance with institutional and international animal regulations. Randomization was conducted and mice were treated by an unblinded manner. Animal protocols were approved by the Institutional Animal Care and Use Committee of Sun Yat-sen University Cancer Center. To evaluate whether knockdown of AMPK $\alpha 1$ could inhibit tumor growth, $1 \times 10^{6}$ RKO or HCT116 cells expressing controlshRNA (sh-NC), AMPK $\alpha 1$-shRNA2 (sh-2), or AMPK $\alpha 1$ shRNA3 (sh-3) were subcutaneously injected into the right flank of the mice $(n=6)$. Tumor volumes were measured on day 7 and every other day from day 12 . The tumor volume was calculated with the equation: $\mathbf{V}\left(\mathrm{mm}^{3}\right)=\mathbf{a} \times \mathbf{b}^{2} / 2$, where $\mathbf{a}$ is the longest diameter and $\mathbf{b}$ is the shortest diameter. Twenty days later, mice were sacrificed and tumors were dissected out and prepared for paraffin-embedded sectioning.

\section{NADPH, GSH, and ATP assays}

The intracellular levels of GSH were measured with the GSH-Glo $^{\text {TM }}$ kit (Promega, WI, USA). Briefly, 8000 cells were incubated in a 96-well plate. The next day, the medium was removed, rinsed twice with PBS and glucose-free medium was added. A total of $100 \mu \mathrm{L}$ of $1 \times \mathrm{GSH}-\mathrm{Glo}^{\mathrm{TM}}$ reagent were then added into the 96-well plate followed by the removal of the medium $12 \mathrm{~h}$ later. With slight shaking, the well was incubated for $30 \mathrm{~min}$ at room temperature. Equal volumes of reconstituted luciferin-detection reagent were then added into each well and samples were incubated for an additional $15 \mathrm{~min}$ at room temperature. Luminescence 
detection in a micro-well reader was then conducted. The intracellular levels of NADPH, total NADP (NADPH + $\mathrm{NADP}^{+}$), and ATP were measured with the NADP/NADPH$\mathrm{Glo}^{\mathrm{TM}}$ kit and the CellTiter-Glo ${ }^{\circ}$ kit (Promega, WI, USA), respectively, according to the manufacturer's instructions.

\section{SiRNA transfection and lentivirus transduction}

Specific siRNA targeting AMPK $\alpha 1$ (Ribobio, Guangzhou, China) were transfected into the indicated cells using Lipofectamine 2000 (Invitrogen, CA, USA) according to the manufacturer's instructions. Target sequences used were as follows: AMPK $\alpha 1$ siRNA1, GAGGAGAGCTATTTG ATTA; AMPK $\alpha 1$ siRNA2, GCAGAAGTATGTAGA GCAA. The cells were harvested $48 \mathrm{~h}$ post transfection for ROS detection and apoptosis analysis or for western blotting to verify and quantify the knockdown efficiency. Stable AMPK $\alpha 1$ and GSR knockdown cells were generated as previously described [43] and the target sequences were as follows:

AMPKo1-shRNA1, CGGCAAAGTGAAGGTTGGCAA ACAT;

AMPK $\alpha 1$-shRNA2, GCTGCACCAGAAGTAATTTCA;

AMPK $\alpha 1$-shRNA3, GACCTCACTTGACTCTTCTCC TGTT;

GSR-shRNA, CCAAGTTGTGAGGGTAAAT.

\section{Assay for reactive oxygen species}

The intracellular levels of ROS were measured with 5-(and6)-Carboxy-2',7'-Dichlorofluorescein Diacetate (DCF-DA). Cells $\left(1 \times 10^{5}\right)$ were plated in a 12 -well plate and were transfected with specific siRNA, cultured with $5 \mathrm{mM}$ glucose or glucose-free medium and were treated with DCFDA for $30 \mathrm{~min}$. The cells were then washed with PBS and collected as single-cell suspensions. Cell fluorescence was detected by flow cytometry.

\section{Statistics}

All data are presented as the mean $\pm \mathrm{SD}$. The immunohistochemical analysis about CRC tissue samples was performed by a blinded manner. To compare the significant differences of more than two groups, one-way ANOVA and Newman-Keuls multiple comparison tests were used. Other statistical analyses were performed using the unpaired Student's $t$-test (GraphPad Prism). For survival evaluation, the Kaplan-Meier method was used to investigate the correlation between variables and overall survival, while a logrank test was employed to compare survival curves. We also used a Cox regression model to perform the univariate and multivariate survival analyses. All of the above methods for survival analysis were performed with the survival package of R software and the other statistical tests were performed with $\mathrm{R}$ software version 3.1.0. The variance between the groups that are statistically compared is similar. Statistical significance was set at 0.05 .

Acknowledgements This study was supported, in part, by the National Key Research and Development Program of China (2017YFC1308900, 2018YFC1313300), Natural Science Foundation of Guangdong Province (2014A030312015, 2017A030313485); National Natural Science Foundation of China (81872011, 81572392); Science and Technology Program of Guangdong (2019B020227002), Science and Technology Program of Guangzhou (201904020046, 201803040019, 201704020228), Guangzhou Health and Medical Collaborative Innovation Project (201704020220), and the Sun Yat-sen University Clinical Research 5010 Program (2018014). FW is the Young Physician Scientist Program of Sun Yat-sen University Cancer Center.

\section{Compliance with ethical standards}

Conflict of interest The authors declare that they have no conflict of interest.

Publisher's note Springer Nature remains neutral with regard to jurisdictional claims in published maps and institutional affiliations.

Open Access This article is licensed under a Creative Commons Attribution 4.0 International License, which permits use, sharing, adaptation, distribution and reproduction in any medium or format, as long as you give appropriate credit to the original author(s) and the source, provide a link to the Creative Commons license, and indicate if changes were made. The images or other third party material in this article are included in the article's Creative Commons license, unless indicated otherwise in a credit line to the material. If material is not included in the article's Creative Commons license and your intended use is not permitted by statutory regulation or exceeds the permitted use, you will need to obtain permission directly from the copyright holder. To view a copy of this license, visit http://creativecommons. org/licenses/by/4.0/.

\section{References}

1. Schreuders EH, Ruco A, Rabeneck L, Schoen RE, Sung JJ, Young GP, et al. Colorectal cancer screening: a global overview of existing programmes. Gut. 2015;64:1637-49.

2. Li M, Gu J. Changing patterns of colorectal cancer in China over a period of 20 years. World J Gastroenterol. 2005;11:4685-88.

3. Sung JJ, Ng SC, Chan FK, Chiu HM, Kim HS, Matsuda T, et al. An updated Asia Pacific consensus recommendations on colorectal cancer screening. Gut. 2015;64:121-32.

4. Chen W, Zheng R, Zeng H, Zhang S. The updated incidences and mortalities of major cancers in China, 2011. Chin J cancer. 2015;34:502-7.

5. Zhou Q, Li K, Lin GZ, Shen JC, Dong H, Gu YT, et al. Incidence trends and age distribution of colorectal cancer by subsite in Guangzhou, 2000-2011. Chin J cancer. 2015;34:358-64.

6. Lee YC, Lee YL, Chuang JP, Lee JC. Differences in survival between colon and rectal cancer from SEER data. PloS One. 2013;8:e78709.

7. O'Connell JB, Maggard MA, Ko CY. Colon cancer survival rates with the new American Joint Committee on cancer sixth edition staging. J Natl Cancer Inst. 2004;96:1420-25.

8. Brenner H, Kloor M, Pox CP. Colorectal cancer. Lancet. 2014;383:1490-502. 
9. Hari DM, Leung AM, Lee JH, Sim MS, Vuong B, Chiu CG, et al. AJCC cancer staging manual 7th edition criteria for colon cancer: do the complex modifications improve prognostic assessment? J Am Coll Surg. 2013;217:181-90.

10. Jeon SM, Hay N. The dark face of AMPK as an essential tumor promoter. Cell Logist. 2012;2:197-202.

11. Hardie DG, Ross FA, Hawley SA. AMPK: a nutrient and energy sensor that maintains energy homeostasis. Nat Rev Mol Cell Biol. 2012;13:251-62.

12. Mihaylova MM, Shaw RJ. The AMPK signalling pathway coordinates cell growth, autophagy and metabolism. Nat Cell Biol. 2011;13:1016-23.

13. Faubert B, Boily G, Izreig S, Griss T, Samborska B, Dong Z, et al. AMPK is a negative regulator of the Warburg effect and suppresses tumor growth in vivo. Cell Metabol. 2013;17:113-24.

14. Shackelford DB, Shaw RJ. The LKB1-AMPK pathway: metabolism and growth control in tumour suppression. Nat Rev Cancer. 2009;9:563-75.

15. AMPK can suppress tumorigenesis and the Warburg effect. Cancer Discov. 2013; 3: OF15. https://cancerdiscovery.aacrjourna 1s.org/content $/ 3 / 3 / \mathrm{OF} 15$.long.

16. Faubert B, Vincent EE, Poffenberger MC, Jones RG. The AMPactivated protein kinase (AMPK) and cancer: many faces of a metabolic regulator. Cancer Lett. 2015;356:165-70.

17. Jeon SM, Hay N. The double-edged sword of AMPK signaling in cancer and its therapeutic implications. Arch Pharm Res. 2015;38:346-57.

18. Jeon SM, Chandel NS, Hay N. AMPK regulates NADPH homeostasis to promote tumour cell survival during energy stress. Nature. 2012;485:661-5.

19. Saito Y, Chapple RH, Lin A, Kitano A, Nakada D. AMPK protects leukemia-initiating cells in myeloid leukemias from metabolic stress in the bone marrow. Cell Stem Cell. 2015;17:585-96.

20. Santoro V, Jia R, Thompson H, Nijhuis A, Jeffery R, Kiakos K, et al. Role of reactive oxygen species in the abrogation of oxaliplatin activity by cetuximab in colorectal cancer. J Natl Cancer Inst. 2016;108:djv394.

21. Shaw RJ. AMPK keeps tumor cells from starving to death. Cell Stem Cell. 2015;17:503-4.

22. Svensson RU, Shaw RJ. Cancer metabolism: tumour friend or foe. Nature. 2012;485:590-1.

23. Goh V, Engledow A, Rodriguez-Justo M, Shastry M, Peck J, Blackman G, et al. The flow-metabolic phenotype of primary colorectal cancer: assessment by integrated 18F-FDG PET/perfusion CT with histopathologic correlation. J Nucl Med. 2012;53:687-92.

24. Fu X, Zhao JX, Zhu MJ, Foretz M, Viollet B, Dodson MV, et al. AMP-activated protein kinase alpha1 but not alpha2 catalytic subunit potentiates myogenin expression and myogenesis. Mol Cell Biol. 2013;33:4517-25.

25. Phoenix KN, Devarakonda CV, Fox MM, Stevens LE, Claffey KP. AMPKalpha2 suppresses murine embryonic fibroblast transformation and tumorigenesis. Genes Cancer. 2012;3:51-62.

26. Kato K, Ogura T, Kishimoto A, Minegishi Y, Nakajima N, Miyazaki M, et al. Critical roles of AMP-activated protein kinase in constitutive tolerance of cancer cells to nutrient deprivation and tumor formation. Oncogene. 2002;21:6082-90.

27. Huang FY, Chiu PM, Tam KF, Kwok YK, Lau ET, Tang MH, et al. Semi-quantitative fluorescent PCR analysis identifies
PRKAA1 on chromosome 5 as a potential candidate cancer gene of cervical cancer. Gynecol Oncol. 2006;103:219-25.

28. Zhao G, Zhang JG, Liu Y, Qin Q, Wang B, Tian K, et al. miR$148 \mathrm{~b}$ functions as a tumor suppressor in pancreatic cancer by targeting AMPKalpha1. Mol Cancer Ther. 2013;12:83-93.

29. Chiu YC, Shieh DC, Tong KM, Chen CP, Huang KC, Chen PC, et al. Involvement of AdipoR receptor in adiponectin-induced motility and alpha2beta1 integrin upregulation in human chondrosarcoma cells. Carcinogenesis. 2009;30:1651-9.

30. Bhandaru M, Martinka M, Li G, Rotte A. Loss of AMPKalpha1 expression is associated with poor survival in melanoma patients. J Invest Dermatol. 2014;134:1763-6.

31. Trachootham D, Alexandre J, Huang P. Targeting cancer cells by ROS-mediated mechanisms: a radical therapeutic approach? Nat Rev Drug Disco. 2009;8:579-91.

32. Yuan S, Lu Y, Yang J, Chen G, Kim S, Feng L, et al. Metabolic activation of mitochondria in glioma stem cells promotes cancer development through a reactive oxygen species-mediated mechanism. Stem Cell Res Ther. 2015;6:198.

33. Lu SC. Regulation of glutathione synthesis. Mol Asp Med. 2009;30:42-59.

34. Ma MZ, Chen G, Wang P, Lu WH, Zhu CF, Song M, et al. Xcinhibitor sulfasalazine sensitizes colorectal cancer to cisplatin by a GSH-dependent mechanism. Cancer Lett. 2015;368:88-96.

35. Trachootham D, Zhou Y, Zhang H, Demizu Y, Chen Z, Pelicano $\mathrm{H}$, et al. Selective killing of oncogenically transformed cells through a ROS-mediated mechanism by beta-phenylethyl isothiocyanate. Cancer cell. 2006;10:241-52.

36. Yun J, Mullarky E, Lu C, Bosch KN, Kavalier A, Rivera K, et al. Vitamin C selectively kills KRAS and BRAF mutant colorectal cancer cells by targeting GAPDH. Science. 2015;350:1391-6.

37. Hoffman NJ, Parker BL, Chaudhuri R, Fisher-Wellman KH, Kleinert M, Humphrey SJ, et al. Global phosphoproteomic analysis of human skeletal muscle reveals a network of exercise-regulated kinases and AMPK substrates. Cell Metabol. 2015;22:922-35.

38. Yu K, Zhang Q, Liu Z, Zhao Q, Zhang X, Wang Y, et al. qPhos: a database of protein phosphorylation dynamics in humans. Nucleic Acids Res. 2019;47:D451-8.

39. Chen DL, Wang ZQ, Zeng ZL, Wu WJ, Zhang DS, Luo HY, et al. Identification of microRNA-214 as a negative regulator of colorectal cancer liver metastasis by way of regulation of fibroblast growth factor receptor 1 expression. Hepatology. 2014;60:598-609.

40. Liu S, Umezu-Goto M, Murph M, Lu Y, Liu W, Zhang F, et al. Expression of autotaxin and lysophosphatidic acid receptors increases mammary tumorigenesis, invasion, and metastases. Cancer cell. 2009;15:539-50.

41. Ju HQ, Li H, Tian T, Lu YX, Bai L, Chen LZ, et al. Melatonin overcomes gemcitabine resistance in pancreatic ductal adenocarcinoma by abrogating nuclear factor-kappaB activation. J Pineal Res. 2016;60:27-38.

42. Bi HC, Pan YZ, Qiu JX, Krausz KW, Li F, Johnson CH, et al. Nmethylnicotinamide and nicotinamide $\mathrm{N}$-methyltransferase are associated with microRNA-1291-altered pancreatic carcinoma cell metabolome and suppressed tumorigenesis. Carcinogenesis. 2014;35:2264-72.

43. Zeng ZL, Luo HY, Yang J, Wu WJ, Chen DL, Huang P, et al. Overexpression of the circadian clock gene Bmal1 increases sensitivity to oxaliplatin in colorectal cancer. Clin Cancer Res. 2014;20:1042-52. 